\title{
A Second Order Delta-Sigma Control of Dielectric Charge for Contactless Capacitive MEMS
}

\author{
S. Gorreta ${ }^{1}$, J. Pons-Nin ${ }^{1}$, E. Blokhina ${ }^{2}$ and M. Dominguez-Pumar ${ }^{1}$ \\ ${ }^{1}$ Micro and Nano Technologies Group, Electronic Engineering Department, Universitat Politècnica de Catalunya, Spain \\ ${ }^{2}$ School of Electrical, Electronic and Communications Engineering, University College Dublin, Ireland
}

\begin{abstract}
This letter introduces a new second-order deltasigma method for control of the dielectric charge in contactless capacitive MEMS. This method improves one previously proposed by the authors, providing second-order quantization noise shaping and allowing to avoid the plateaus typical of first-order strategies. The feasibility and the features of the new method are demonstrated both experimentally and through simulations.
\end{abstract}

Index Terms-Dielectric charging control, MEMS reliability

\section{INTRODUCTION}

Dielectric charging is a serious reliability problem that slows down the use of electrostatic MEMS in many applications [1]. The horizontal voltage shift of the $C(V)$ curve, $V_{s h}$, is related to the net amount of charge trapped in the dielectric, $Q_{d}$. For MEMS switches, this implies undesired drift in key features like pull-in and pull-out voltages.

Several actuation strategies, based on the opposite effects of bipolar voltages on the charge dynamics, have been proposed to mitigate the effects of dielectric charging [1]-[4]. However, only closed-loop control strategies based on periodical sensing of dielectric charge or its effects can ensure long-term stability of the device features. Moreover, strategies based on absolute measurements of the device capacitance cannot ensure such stability against vertical drifts of the $C(V)$ caused by nonuniform charge distributions, or variations in temperature, humidity, etc. [5], [6].

The control method proposed in [7] addresses the above issues for devices working below pull-in. A "quasi-differential" device capacitance $\Delta C$ provides an indirect measurement of $V_{s h}$, thus of $Q_{d}$, at each sampling time. Depending on whether $\Delta C$ is above or below a threshold value $\Delta C_{t h}$, a specific voltage symbol, BIT0 or BIT1 (see fig. 1), is applied in the next sampling period. The total dielectric charge is kept almost constant to a target level $Q_{\text {targ }}$ by dynamical adjustment of the sequence of symbols, or bitstream, applied.

This method is based on $1^{\text {st }}$ order delta-sigma $(\Delta \Sigma)$ modulation. The dielectric works as a leaky charge integrator under two competing mechanisms: charge being injected by the actuation symbols and charge escaping the traps in the material. The $\Delta \Sigma$ modulator generates an actuation bitstream that compensates in average the charge being leaked out of the dielectric so that $Q_{d}=Q_{\text {targ }}$.

However, known issues of $1^{s t}$ order $\Delta \Sigma$ modulators limit the effectiveness of this method. One is the presence of tones in the spectrum of the bitstream (see fig. 18 in [7]), which poses a problem for retrieving real-time information about the charge

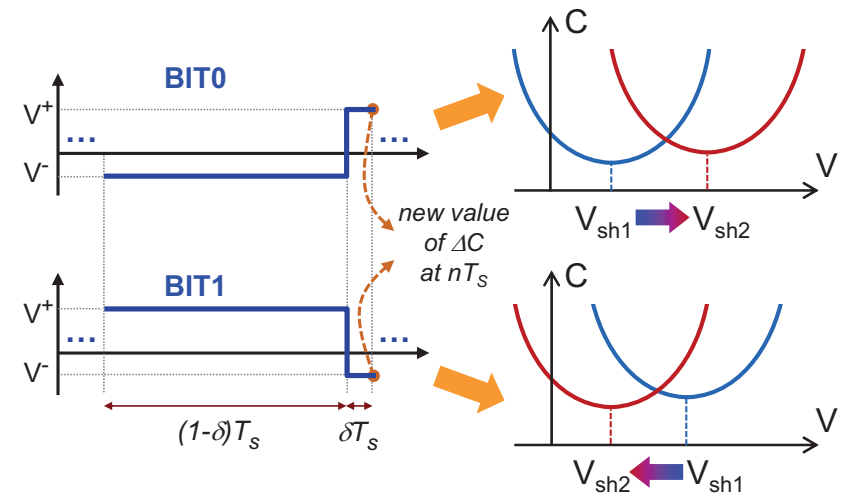

Fig. 1. Bipolar voltage symbols applied [7]. In the MEMS used, BIT0 and BIT1 produce opposite but not symmetrical shifts of the $C(V)$. Capacitances $C^{+}=C\left(V^{+}\right)$and $C^{-}=C\left(V^{-}\right)$are measured at symbol instants $(1-\delta) T_{s}$ and $T_{s}$, and $\Delta C_{n}=\Delta C\left(n T_{s}\right)$ is obtained as $=C^{+}-C^{-}$.

status. Another issue is the presence of a Devil's Staircase fractal (see figs. 11 to 13 in [8]), a typical effect when leaky integrators are used. The fractal plateaus are "dead zones" that cannot be observed or controlled, thus hindering the possibility of having a good charge control in certain cases.

\section{SECOND ORDER CONTROL OF DIELECTRIC CHARGE}

The $2^{n d}$ order method proposed here improves the previous $1^{\text {st }}$ order one. It works as shown in fig. 2(a): a voltage symbol, BIT0 or BIT1, is applied in the current sampling period $T_{s}$ and the quasi-differential capacitance $\Delta C_{n}$ is obtained at the end of the symbol, fig. 1 . Then the error signal $\Delta C_{n}-\Delta C_{t h}$ is integrated numerically and the sign of the result decides the symbol applied in the next sampling period.

For slow time evolution of the charge leakage $\beta$, the analysis of the equivalent sampled circuit (fig. 2(b)) yields the following governing equations:

$$
\begin{array}{r}
\Delta Q_{n+1}=\Delta Q_{n}+\beta-b_{n} \\
w_{n+1}=w_{n}+\Delta Q_{n}
\end{array}
$$

where $w_{n}$ is the integrated charge error (thus $w_{0}=0$ ) and $b_{n}=\operatorname{sign}\left(w_{n}+\Delta Q_{n}\right)$ is the bitstream sequence. This denotes a $2^{\text {nd }}$ order $\Delta \Sigma$ modulator with two-cascaded charge integrators. The first is the MEMS dielectric itself, which acts as a low-pass filter with typical $1^{\text {st }}$ order noise shaping [7]. The second integrator improves noise rejection at low frequencies, thus providing $2^{\text {nd }}$ order noise shaping. Fig. 3, taken from 
a)

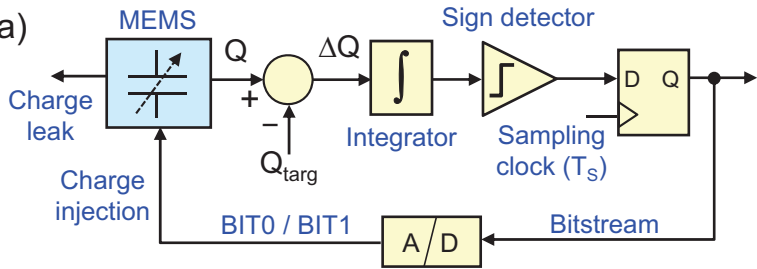

b)

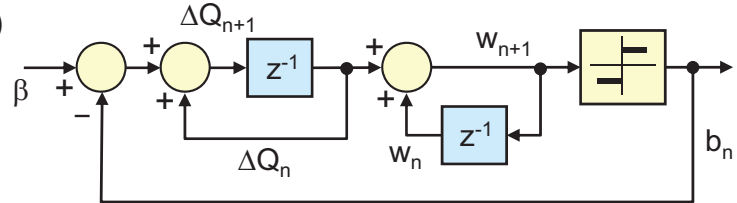

Fig. 2. Block diagram (a) and equivalent sampled circuit (b) of the $2^{\text {nd }}$ order control method. In practice the sensing variables are quasi-differential capacitances, but equivalent charges are used here instead.

experimental data, illustrates how the second integrator works. Note that the behaviours at the output of the first (a) and at the output of the second (b) integrators are similar to those typical in $2^{\text {nd }}$ order modulators [9].
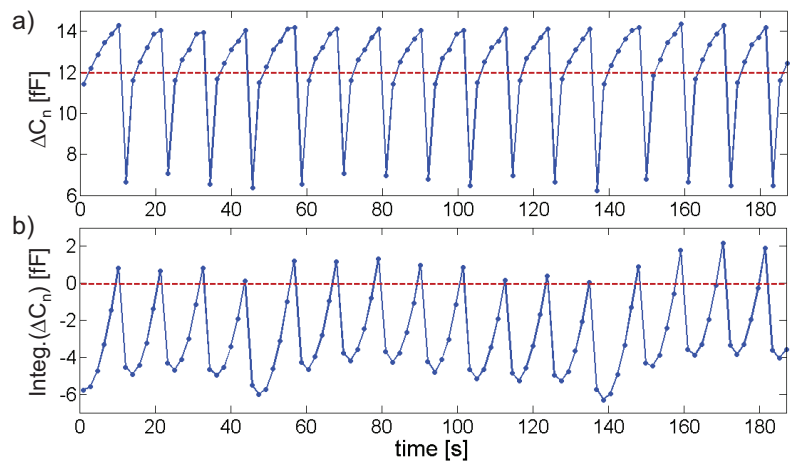

Fig. 3. (a) Sample sequences taken from experiments (a) at the output of the first integrator, $\Delta C_{n}$, and (b) at the output of the second-numerical integrator. The threshold capacitance $\Delta C_{t h}$ is $12 \mathrm{fF}$.

\section{RESULTS AND DISCUSSION}

The $2^{\text {nd }}$ order control was implemented using a precision impedance meter controlled from a computer, see fig. 4. Two MEMS devices, Dev-1 and Dev-2, fabricated with the standard multi-user PolyMUMPS ${ }^{\circledR}$ process from MEMSCAP ${ }^{\circledR}$ were used. In Dev-1 the moveable electrode is a 500x $500 \mu \mathrm{m}^{2}$ goldcovered polysilicon plate, suspended over a $2.75 \mu \mathrm{m}$ air gap and a $0.6 \mu \mathrm{m}$ thick silicon nitride layer deposited on the dopedsilicon substrate, which is the fixed electrode; pull-in voltage is $14 \mathrm{~V}$. Dev-2 has similar structure, but $360 \times 360 \mu \mathrm{m}^{2}$ area and $24 \mathrm{~V}$ pull-in. The charging dynamics of both devices were characterized using the multi-exponential models and fitting procedure from [7]. The devices were fabricated in batches widely separated in time, and they exhibit very different charging and discharging rates, with the fastest time constants being 13 minutes for Dev-1 and $335 \mathrm{~ms}$ for Dev-2.

The first experiment focused on the feasibility of both control methods to obtain a given sequence of target values of

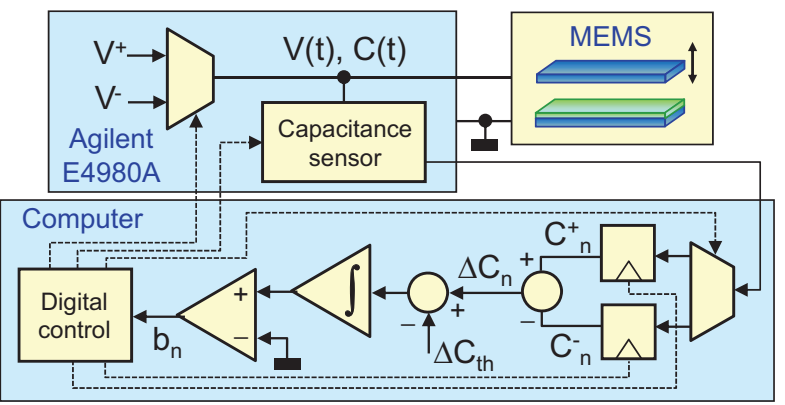

Fig. 4. Experimental set-up. The instrument applies the voltage symbols and measures the MEMS capacitance, while a computer program processes the capacitance data and decides the next symbol.

$V_{s h}$, thus of $Q_{d}$. Fig. 5 shows the results obtained when each method is applied to Dev-1 to obtain $V_{s h}=0.5 \mathrm{~V},-0.75 \mathrm{~V}$ and $0 \mathrm{~V}$ in 48-hour intervals. Note that the values of $V_{s h}$ fairly match with the desired ones for both methods. Moreover, once steady-state regimes are reached, the values of $V_{s h}$ are always kept constant.

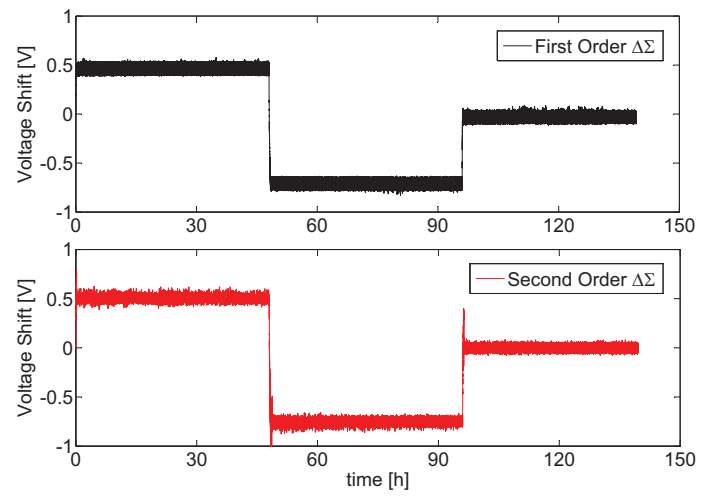

Fig. 5. Results of the control methods applied to Dev-1 to obtain three different values of $V_{s h} . V^{+}=-V^{-}=4 \mathrm{~V}, T_{s}=2.5 \mathrm{~s}, \delta=1 / 5$.

$\Delta \Sigma$ modulators allow to extract real-time information about the behaviour of the input variable by low-pass filtering, or averaging, the control bitstream. Additionally, in our case the net charge trapped in the dielectric $Q_{d}$ can be indirectly monitored from the evolution of $\Delta C_{n}$. Moreover, the charge dynamics models obtained allow to predict the evolution of the control variables for a given device. Then, discrete-time simulations of the evolution of the bitstream average for the experiment of fig. 5 have been performed. Fig. 6 compares experimental bitstream averages with their simulated counterparts. Note that all bitstreams, measured and simulated, reach the same values once in steady-state, but they also exhibit the same behaviour in the transients. The fair results reported in figs. 5 and 6 demonstrate the feasibility of the proposed method to provide an effective control of dielectric charge.

Fig. 7 compares the spectral power densities of the bitstreams obtained from an experiment on which Dev-1 was set to $V_{s h}=-0.5 \mathrm{~V}$ using both control methods. The presence of the additional integrator in the $2^{\text {nd }}$ order method produces noticeable differences. Firstly, the tones around $0.1 \mathrm{~Hz}$ found 


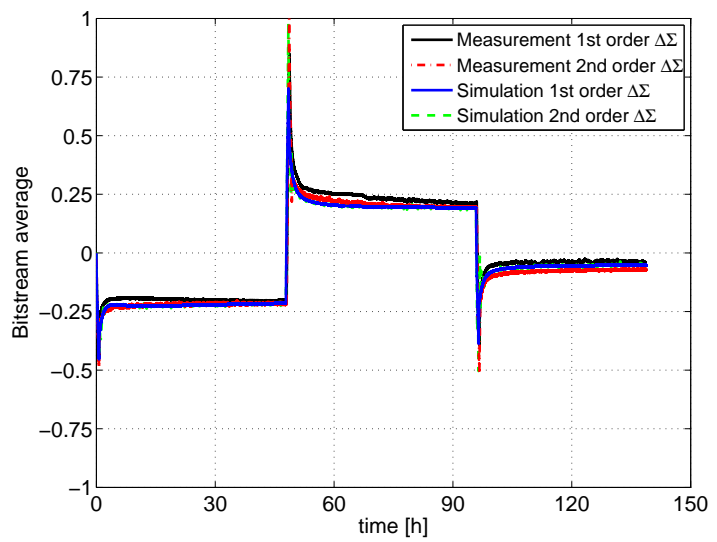

Fig. 6. Comparison between the filtered control bitstreams extracted from the experiment of fig. 5 and the ones obtained from simulations.

in the $1^{\text {st }}$ order method are no longer present in the $2^{\text {nd }}$ order one. Furthermore, the noise at low frequencies becomes considerably reduced and the slope of the quantization noise rolled out of the band of interest clearly increases in the $2^{\text {nd }}$ order case. Let us note that these results strongly resemble those obtained in [10], where $1^{\text {st }}$ and $2^{\text {nd }}$ order thermal $\Delta \Sigma$ modulators for flow sensing applications are compared.

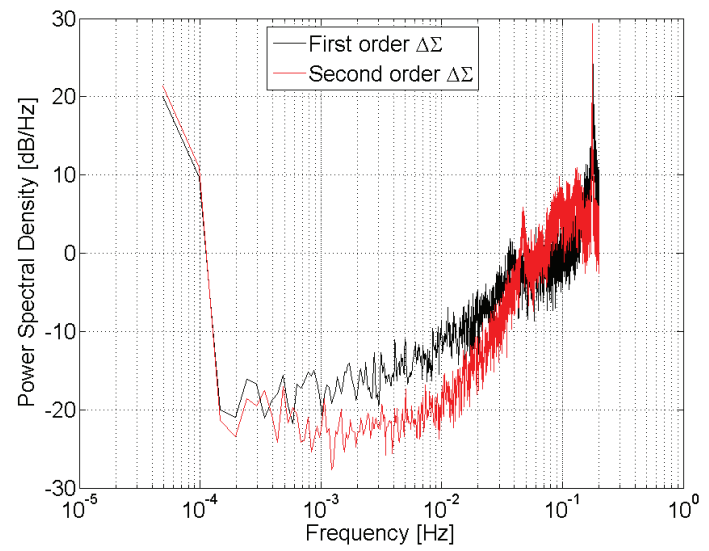

Fig. 7. Power spectrum densities (P-Welch estimation) obtained from 24000 samples of the control bitstream. $1^{\text {st }}$ and $2^{\text {nd }}$ order methods applied to Dev-1 to obtain $V_{s h}=-0.5 \mathrm{~V}$.

The fractal sensitivity of both methods was also checked using Dev-2, which exhibits faster charging dynamics. The experiment consisted of applying real-time charge control to obtain stepped values of $\Delta C_{t h}$, thus of $Q_{\text {targ. }}$ Each step lasted 30 minutes, time enough to achieve stable regimes. The bitstream average obtained in each step is shown in fig. 8. Both simulation and experimental results for the $1^{\text {st }}$ order control show fractal behaviour: the same bitstream average is observed for four different values of $\Delta C_{t h}$ around zero, then fair control leading to a discharged state was not possible in this case. On the other hand, the fractal behaviour is no longer seen and thus the relationship between the output bitstream and the total dielectric charge becomes univocal when using the $2^{\text {nd }}$ order control method.

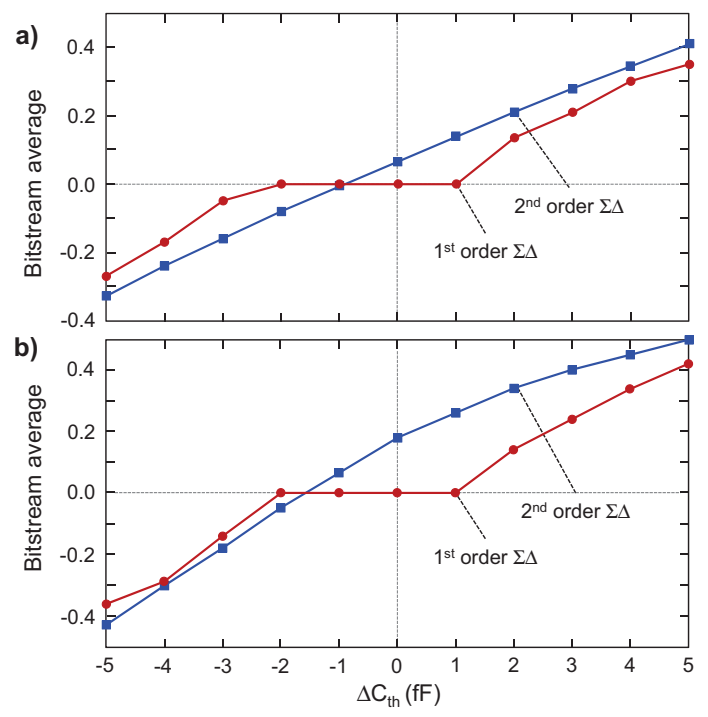

Fig. 8. Simulated (a) and measured (b) bitstream average values obtained with Dev-2 for several values of $\Delta C_{t h}$. Each point of the graph corresponds to a 30 -minute experiment. $V^{+}=-V^{-}=10 \mathrm{~V}, T_{s}=1.7 \mathrm{~s}, \delta=1 / 7$.

\section{CONCLUSIONS}

A new second order method of dielectric charging has been presented. The main advantage is that the quantization noise shaping is second order and that the typical plateaus of first order can be avoided.

\section{ACKNOWLEDGMENT}

This work was supported in part by the Spanish Ministry MINECO under Project TEC2013-48102-C2-1P.

\section{REFERENCES}

[1] W. Van Spengen, "Capacitive RF MEMS switch dielectric charging and reliability: a critical review with recommendations," Micromechanics and Microengineering, J. of, vol. 22, 2012.

[2] T. Ikehashi et al., "An RF MEMS variable capacitor with intelligent bipolar actuation," in Int. Solid-State Circuits Conf., 2008, pp. 581-583.

[3] M. Dominguez-Pumar, D. Lopez, D. Molinero, and J. Pons, "Dielectric charging control for electrostatic MEMS switches," in SPIE Conf. on Defense, Security and Sensing, vol. 7679, pp.1-11, Orlando, 2010.

[4] E. Blokhina, S. Gorreta, D. Lopez, D. Molinero, O. Feely, J. Pons, and M. Dominguez-Pumar, "Dielectric charge control in electrostatic MEMS positioners / varactors," IEEE-JMEMS, vol. 21, pp. 559-573, 2012.

[5] X. Rottenberg et al., "Analytical model of the DC actuation of electrostatic MEMS devices with distributed dielectric charging and nonplanar electrodes," IEEE-JMEMS, vol. 16, pp. 1243-1253, 2007.

[6] A. Jain, S. Palit, and M. A. Alam, "A physics-based predictive modeling framework for dielectric charging and creep in RF MEMS capacitive switches and varactors," IEEE-JMEMS, vol. 21, pp. 420-430, 2012.

[7] S. Gorreta, J. Pons, E. Blokhina, O. Feely, and M. Domínguez-Pumar, "Delta-sigma control of dielectric charge for contactless capacitive MEMS," IEEE-JMEMS, vol. 23, no. 4, pp. 829-841, 2014.

[8] M. Dominguez-Pumar et al., "Real-time characterization of dielectric charging in contactless capacitive MEMS," In Press in Analog Integrated Circuits and Signal Processing, 2015.

[9] P. Aziz, H. Sorensen, and J. Van Der Spiegel, "An overview of sigmadelta control converters," IEEE Signal Processing Magazine, vol. 1, pp. 61-84, 1996.

[10] K. Makinwa and J. Huijsing, "A 2nd order thermal sigma-delta modulator for flow sensing," in IEEE Sensors 2005, Oct, 2005. 